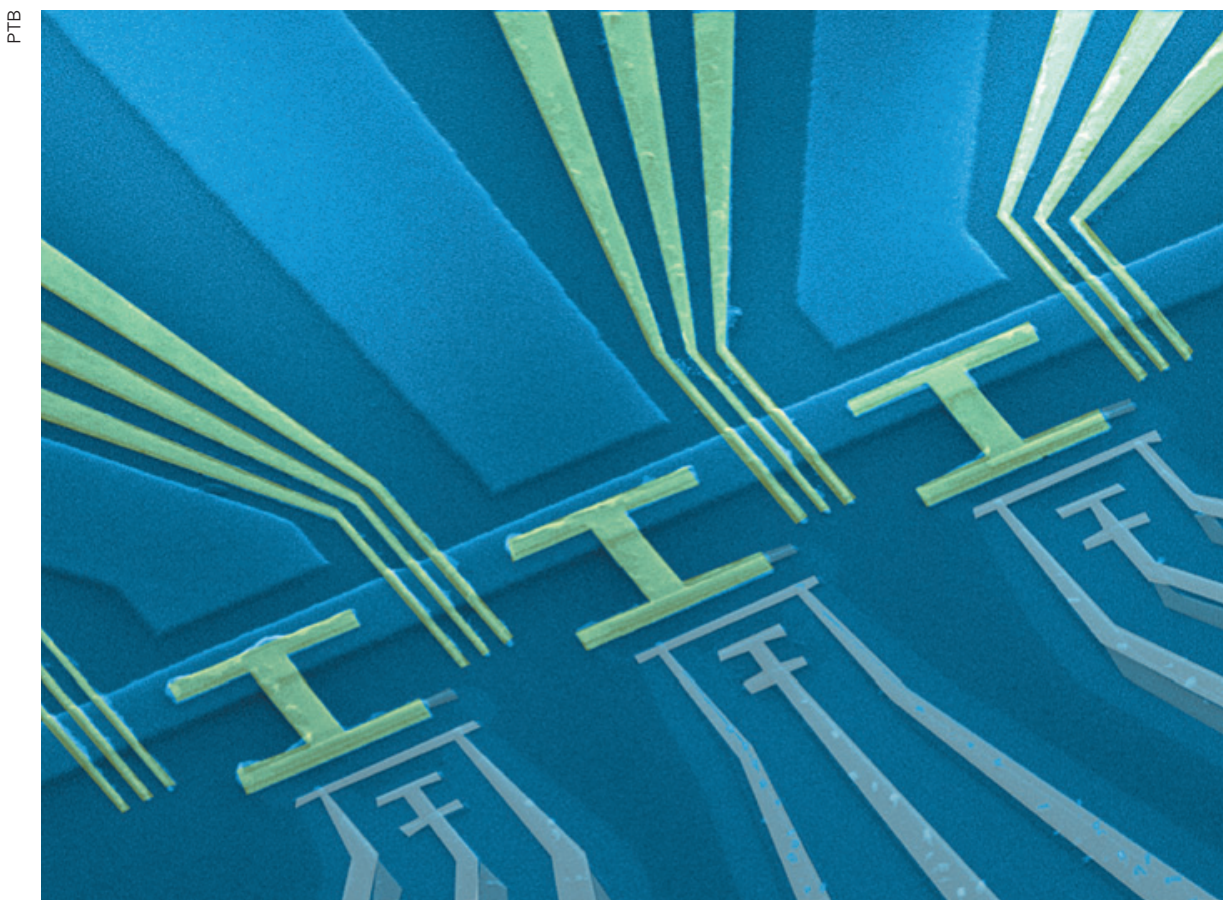

A semiconductor device, seen in a scanning electron micrograph, can measure the flow of single electrons.

METROLOGY

\title{
Ampere to get rational redefinition
}

\section{Single-electron flow measured in bid to overhaul SI base unit.}

\section{BY EUGENIE SAMUEL REICH}

$\mathrm{P}$ hysicists have tracked electrons crossing a semiconductor chip one at a time - an experiment that should at last enable a rational definition of the ampere, the unit of electrical current.

At present, an ampere is defined as the amount of charge flowing per second through two infinitely long wires one metre apart, such that the wires attract each other with a force of $2 \times 10^{-7}$ newtons per metre of length. That definition, adopted in 1948 and based on a thought experiment that can at best be approximated in the laboratory, is clumsy - almost as much of an embarrassment as the definition of the kilogram, which relies on the fluctuating mass of a 125-year-old platinum-and-iridium cylinder stored at the International Bureau of Weights and Measures (BIPM) in Paris.

The new approach, described in a paper ${ }^{1}$ posted onto the arXiv server on 19 December, would redefine the amp on the basis of $e$, a physical constant representing the charge of an electron. Metrologists have long sought such a rational definition. "It's an enormously challenging thing to try and do and it's quite an important paper," says Stephen Giblin, a physicist at the National Physical Laboratory in Teddington, UK.

The result will find favour at a meeting of the BIPM's General Conference on Weights and Measures in November. There, metrologists will discuss a proposal to redefine the ampere, the kilogram and two other standard (SI) units - the mole and the kelvin - in terms of the physical constants $e$, Planck's constant, Avogadro's constant and Boltzmann's constant.

Two other basic SI units, the metre and the second, have already been redefined in terms of two constants: the speed of light and the frequency at which electrons in caesium atoms transition between energy levels.

In the amp experiment ${ }^{1}$, physicist Hans Schumacher of the Federal Institute of Physical and Technical Affairs (PTB) in Braunschweig, Germany, and his colleagues made use of a single-electron pump, a device in which voltage pulses prompt electrons to quantummechanically tunnel across barriers one at a time. The researchers tracked the paths of individual electrons by detecting changes in the electrical charge stored at points between 
the barriers. Primitive electron pumps have existed since 1990, but this is the first time that changes in charge have been detected for each hop of an electron.

The pump transferred just a few dozen electrons per second - slow enough to permit precision measurement and thus to provide proof of principle for redefining the amp. But this is only a first step: the set-up would not be practical for calibrating current-measuring ammeters, which need to run at higher currents. The ultimate goal is to create a 'mise en pratique' - a standard-setting experiment that can be reproduced in any lab to calibrate measurements of current precisely - so the race

"It's
impossible to
say which is
the winning
concept."
is now on to combine Schumacher's validation method with a higher-current pump.

In 2012, Giblin pioneered a semi-

conductor single-electron pump that transferred nearly one billion electrons per second (ref. 2), but he could not track them one by one. Other types of pump include turnstiles, in which electrons tunnel between superconducting wires, and tunnel junctions, in which electrons tunnel between aluminium islands separated by layers of insulating oxide. But these must also be operated at relatively low currents to track single electrons. "It's impossible to say which is the winning concept," says Jukka Pekola, a physicist at Aalto University in Espoo, Finland, who reviewed approaches to redefining the amp in 2013 (ref. 3).

Still, the amp is in good shape for the November meeting, as is the kelvin. The charge of the electron and Boltzmann's constant have both been measured precisely, so both units are ready to be redefined today, says François Piquemal, a physicist at the National Laboratory of Metrology and Testing in Paris.

But the process could be delayed until the 2018 meeting of the BIPM general conference. All four units are intertwined, so the plan is to redefine them all at once, and the kilogram is causing problems. There are two rival approaches to its redefinition: a watt balance, which would balance a test mass against Earth's gravity in terms of electrical power, and a precise count of atoms in a sphere of silicon. The two approaches give slightly different answers. Piquemal says that the differing approaches need to be reconciled before the units can be redefined.

\footnotetext{
1. Fricke, L. et al. Preprint at http://arxiv.org/ abs/1312.5669 (2013).

2. Giblin, S. P. et al. Nature Commun. 3,930 (2012).

3. Pekola, J. P. et al. Rev. Mod. Phys. 85, 14211472 (2013)
}

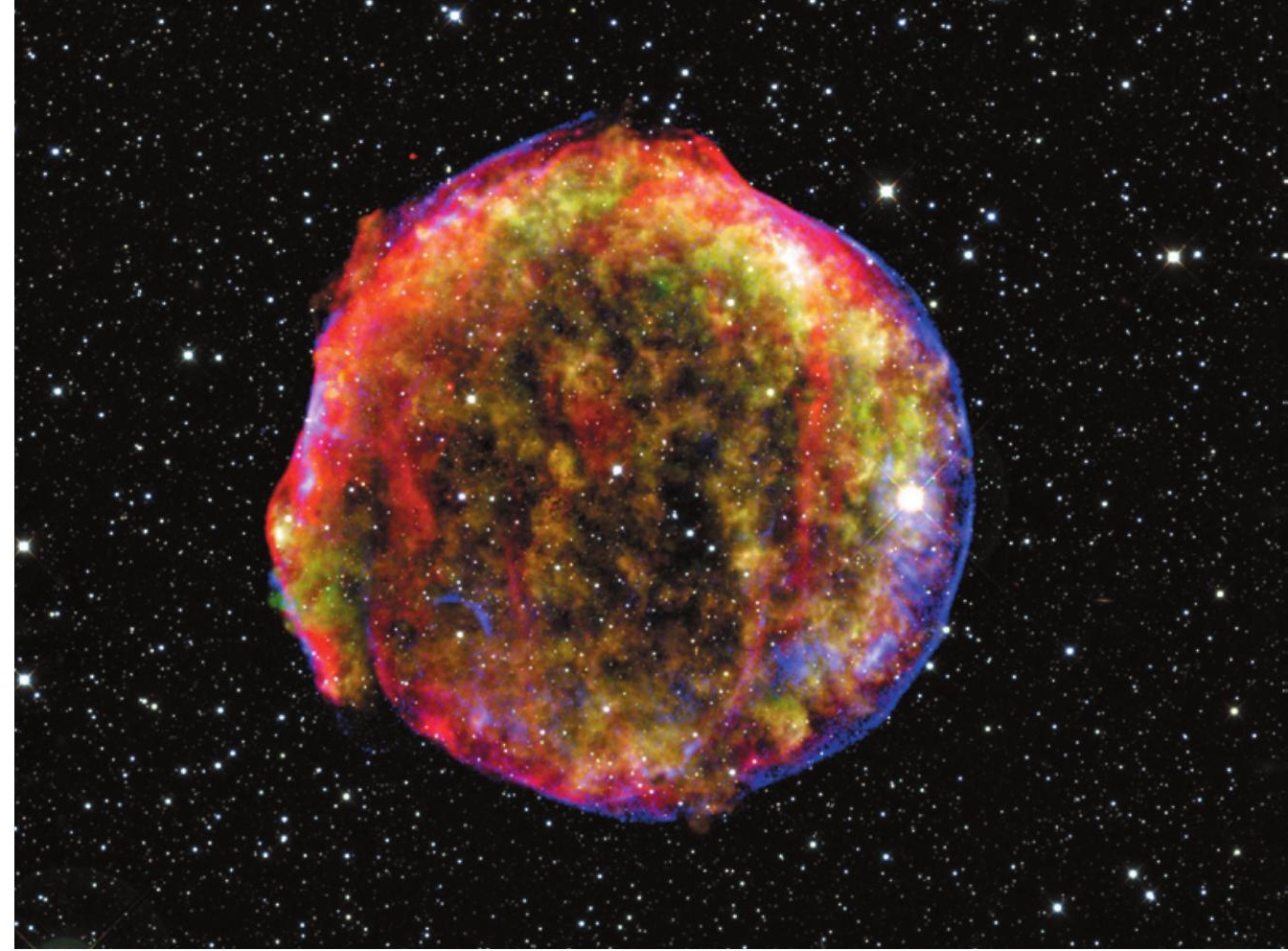

This remnant of Tycho's supernova was created by a type la supernova in 1572 .

\section{ASTROPHYSICS}

\section{Kepler clue to \\ supernova puzzle}

\section{Two white dwarfs favoured as precursors of type Ia supernovae.}

\section{BY RON COWEN}

$\mathrm{T}$ They are cosmic detonations that briefly outshine the light of entire galaxies. And they were a crucial tool in the discovery of dark energy, the force that is accelerating the expansion of the Universe. Yet the process that gives rise to type Ia supernovae has remained mysterious.

Now, light from two of these stellar explosions has been captured in finer temporal detail than ever before, and the data are adding weight to an emerging view: that the explosions result from the merger of two white dwarfs, the burnt-out, Earth-sized remnants of Sun-like stars. The finding erodes a longstanding view that type Ia supernovae arise from a single white dwarf accruing material from an ordinary companion star, either a Sunlike star or an elderly, bloated red giant.

The data have come from an unlikely source: NASA's Kepler mission, the space telescope that searched for alien planets by staring at some 150,000 stars in nearby reaches of the Milky Way. Distant galaxies also lurk in the telescope's field of view, and its ability to collect data every 30 minutes, along with its sensitivity to tiny changes in brightness, made it ideal for recording the rise and fall of light emitted during supernovae.

Robert Olling, an astronomer at the University of Maryland in College Park, was lucky enough to find two type Ia supernovae after a two-year survey of some 400 galaxies in Kepler's field. He reported them on 8 January at a meet ing of the American Astronomical Society near Washington DC. "As a technical tour de force, it's really cool to use Kepler for more than it was intended," says Robert Kirshner, an astronome at the Harvard-Smithsonian Center for Astrophysics in Cambridge, Massachusetts.

The exceptional smoothness of the Kepler data is helping researchers to distinguish between the two competing explosion scenarios. Both require that a white dwarf takes on material from a companion object until the pressure ignites a runaway thermonuclear explosion. But in the ordinary com panion model, the expanding shell of material from the white dwarf would ram into the normal star, generating extra heat and light that would show up as a bump in the first days of a supernova's brightening. No such bump exists in Olling's data.

That essentially rules out all red giant companions, says Olling, because these larger stars would result in a sizeable bump. But the data could still be compatible with smaller, more 